\title{
A shortcut method for sexing juvenile European sea bass, Dicentrarchus labrax L.
}

\author{
Bruno Menu ${ }^{1}$, Stefano Peruzzi ${ }^{1,2}$, AlainVergnet $^{1}$, Marie-OdileVidal $^{1}$ \& Béatrice Chatain $^{1}$
}

1 : IFREMER, Laboratoire de Recherche Piscicole de Méditerranée, Palavas-les-Flots.

2 : Norwegian College of Fishery Science, University of Tromsø, Norway

*: Corresponding author: Bruno Menu. IFREMER, Laboratoire de Recherche Piscicole de Méditerranée, Chemin de Maguelone, F-34250 Palavas-les-Flots, France. E-mail: Bruno.Menu@ifremer.fr

\begin{abstract}
:
The objective of this study was to develop a rapid and effective method of sexing juvenile sea bass with minimum labour and material. For this aim, the gonad squash mount technique was applied along with macroscopic techniques for sexing a large number of experimental fish at the age of 215-275 days post fertilisation (p.f.). At this age, 90\% of the 3894 fish could be unambiguously sexed by macroscopic examination of their gonads, whereas the remaining proportion was identified using squash mount preparations (10\%). The accuracy of the observations was measured up to classical histology procedures. Undifferentiated fish accounted for $0.1 \%$ of the total population only. The application of the squash mount technique holds out the prospect of considerable improvement in the efficiency and rapidity of current sexing techniques in sea bass.
\end{abstract}




\section{Introduction}

The European sea bass (Dicentrarchus labrax L.) is a marine aquaculture species of major economic interest in Europe but it is recognised that the skewed sex-ratio in favour of males observed under culture conditions, and their early maturation compared to females still represent important drawbacks for commercial production (Zanuy et al., 2001). Although significant contributions have already been made in this field, much work is still concerned with sex control methods in this species (Pavlidis et al., 2000; Zanuy et al., 2001; Koumoundourous et al., 2003). Genetic and physiological approaches generally require the set-up of mid-term or long-term studies and considerable amount of work in sexing juveniles or adult fish. Sexually mature sea bass can be recognised macroscopically by their gonads according to the classification of Barnabé (1976). To date, the only method applied for sexing smaller fish is through histological sections only. Although effective, this long-established technique requires considerable amount of time, labour and material, and may not be practical for extensive experimental work. Therefore, an identification of juvenile gonad tissues with minimum labour would be a significant contribution to the development of rapid and reliable sexing procedures in this species.

In this work, we investigated the use of squash mount preparations as a shortcut method for sexing juvenile sea bass whose phenotypic sex is not yet recognisable using macroscopic criteria. Histology procedures were used to confirm the accuracy of all observations. The implications and potential applications of these results are discussed.

\section{Materials and Methods}

Fish originated from groups reared under standard culture conditions at IFREMER Palavas-les-Flots (Chatain et al., 1999). These groups were all composed of 24 
families of fish obtained by full factorial cross ( 6 sires by 4 dams). During the ongrowing phase $n=3894$ fish were sampled at the age of $215-275$ days p.f. and over a period of 2 months. At each sampling, fish were sacrificed by overdose of anaesthetic (2-Phenoxy-ethanol, $500 \mathrm{ppm})$. They were then measured ( $\mathrm{mm}$, total length) and weighed $(\mathrm{g})$ to the nearest 0.01 . Phenotypic sex was first determined, whenever possible, by macroscopic examination of the gonads (Barnabé, 1976). Gonads were then sampled and examined directly by studying wet-squash preparations. For this use, the gonads were removed using fine forceps, mounted on a microscope slide and stained according to Guerrero and Shelton (1974). Briefly, the gonad tissue was placed on a slide, covered with few drops of aceto-carmine and squashed with a cover slip. The slide was then examined under a compound microscope using magnifications of 25 to 50x. For coupled squash and histological examinations, the gonads of 30 randomly chosen fish of this group were dissected, one fresh gonad was prepared and observed as above, while the other gonad was fixed in Bouin-Hollande and treated using standard histological procedures. Gonads were longitudinally sectioned to a thickness of $5 \mu \mathrm{m}$ and stained with erythrosine orange $\mathrm{G}$ and toluidine blue. All mounts were observed and photographed through a Zeiss photomicroscope.

\section{Results}

At 215-275 days p.f., the 3894 fish measured from 109 to $227 \mathrm{~mm}$ (Total Length, TL) and weighed $11-122 \mathrm{~g}$. Their weight class distribution is reported in Fig 1a. The most representative classes were $31-40 \mathrm{~g}$ and $41-50 \mathrm{~g}$ accounting for $29 \%$ and $28 \%$ of the population respectively. At this age, most of the fish (90\%) could be unambiguously sexed by macroscopic examination of their gonads. The remaining $10 \%$ was identified using wet squash preparations. These fish ranged from 109-195 mm (TL) and weighed 11-79 g. Only 4 out of 3894 fish $(0.1 \%)$ did not show distinctive male or female characters and could not be identified by either of the above methods. These fish were 
later classified as undifferentiated at histological level. The percentage of fish sexed by macroscopic, squash and histology examinations in each weight class is reported in Fig $1 b$.
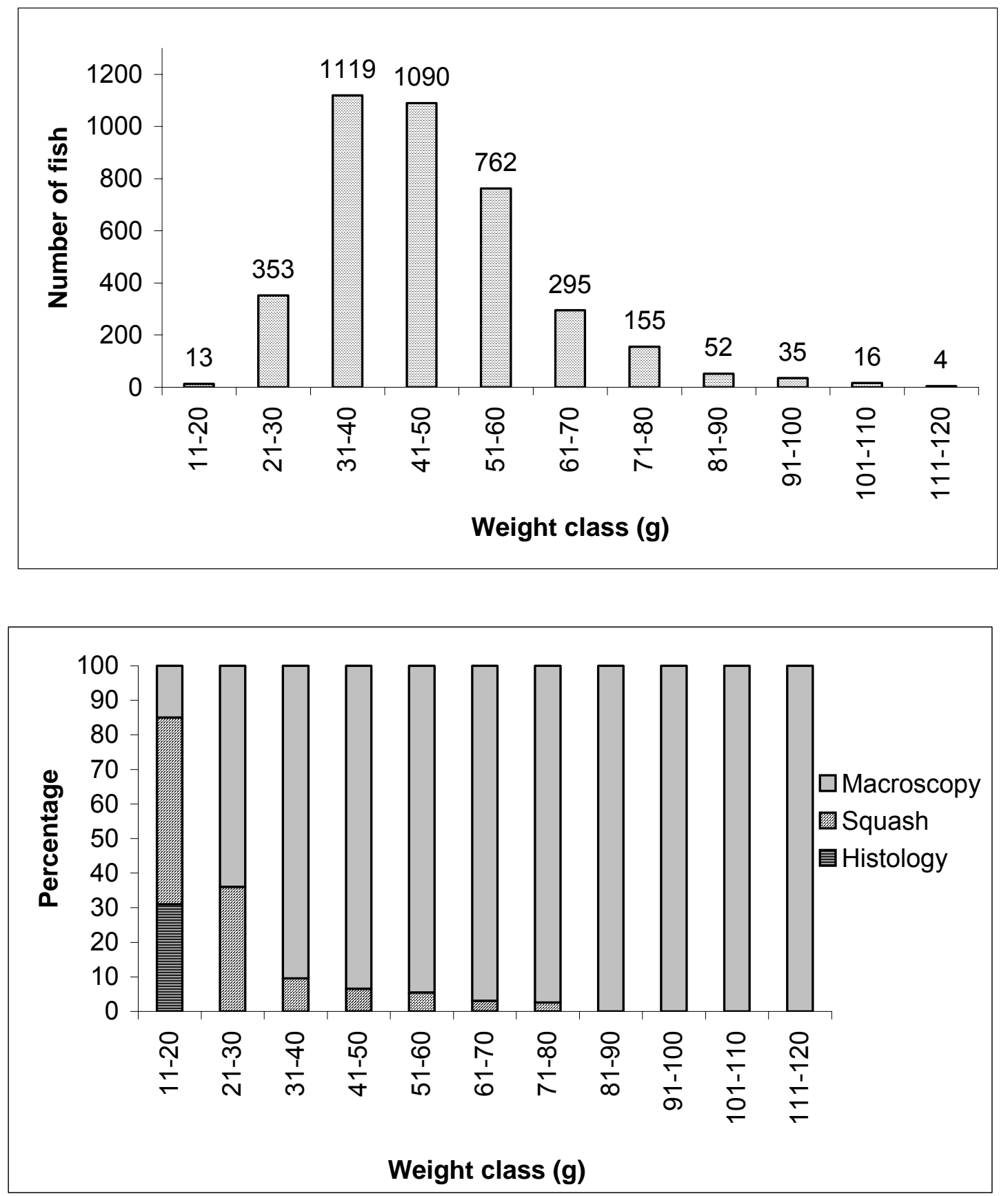

Figure 1 (a) Weight class distribution of fish $(n=3894)$ analysed at the age of $215-275$ days p.f. Superscript values represent the number of fish in each class. (b) Percentages of fish sexed by macroscopic, squash and histological methods in each weight class. 
Representative wet-squash and histology preparations obtained from a female and a male fish are presented in Fig 2. With both methods, ovarian tissues could be easily identified by the presence of large numbers of previtellogenic oocytes (Fig. 2a,b), whereas male germinal tissues were typically arranged in well-developed testicular tubules (Fig. 2c,d). Generally, all fish had differentiated into one sex or the other and the overall sex ratio was skewed in favour of males $(64 \%)$.
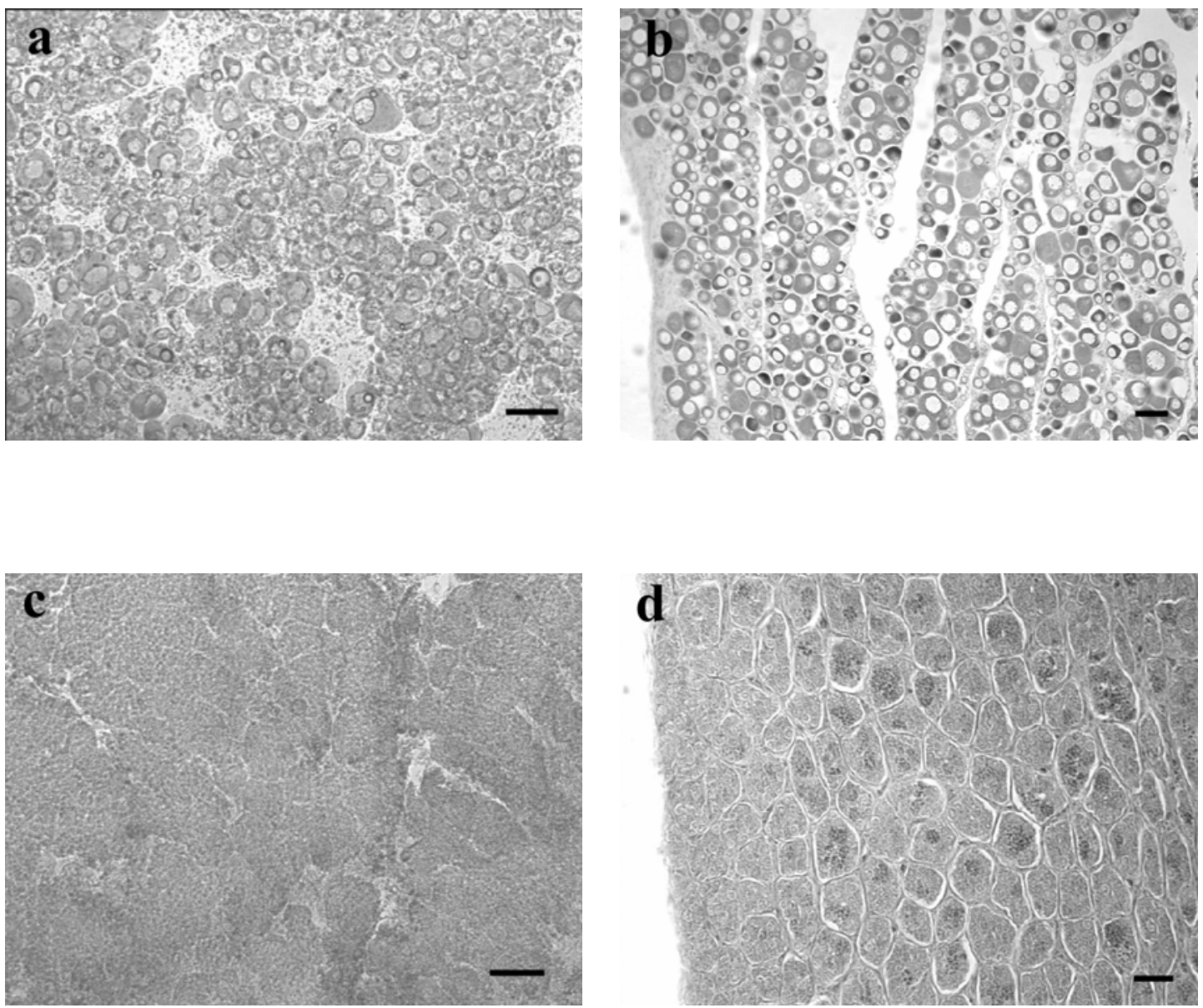

Figure 2 Coupled wet squash preparations $(a, c)$ and histological sections $(b, d)$ of female $(a, b)$ and male (c,d) seabass gonads. (a) Squashed ovary containing different sizes of pre-vitellogenic oocytes. (b) Longitudinal section of the other ovary mostly filled with pre-vitellogenic oocytes. (c) Typical squash preparation of male germinal tissue arranged in testicular lobules. (d) Longitudinal section of the same tissue showing the well developed testicular lobules. Scale bars $=50 \mu \mathrm{m}$. 


\section{Discussion}

The squash technique proved to be a rapid and reliable means of determining the sex of juvenile sea bass whose phenotypic sex is not yet discernible using macroscopic criteria. The accuracy of the observations was confirmed by comparing wet-squash preparations and histological sections from the same individuals. This technique was originally developed on tilapia (Oreochromis aureus) and bluegill (Lepomis macrochirus) by Guerrero and Shelton (1974), but later applied within tilapias only (Abucay et al., 1999, Baras et al., 2001).

In sea bass, Pavlidis et al. (2000) used histological procedures to sex approx. 1000 specimens exposed to different rearing temperatures. Histological analyses were also employed for the sex determination of variable proportions of fish by other authors (Gorshkov et al., 1999; Koumoundourous et al., 2003). In the present work, the majority of fish could be sexed macroscopically and the others, including specimens as small as 18-20 g, could be unambiguously determined by squash mounts. At $215-275$ days p.f., the gonads, while generally small, were sufficiently developed to sex 370 fish beyond the above weight by the presence of developing oocytes or distinctive testicular structures without requiring further histological examination. At this age, sexually undifferentiated gonads were very scarce. This is in general agreement with Saillant and co-workers (In press), who found that distinctive testicular or ovarian differentiation is completed in sea bass beyond the age of 250 days p.f. or standard length of $12 \mathrm{~cm}$. The same authors also outlined that the proportion of females recorded at 168 days p.f. did not vary in subsequent samplings, suggesting that undifferentiated fish found already at this age can develop towards males only. Accordingly, the rare undifferentiated fish observed in the present work were most likely immature males.

A potential advantage of the squash method that might apply to both research and the aquaculture industry is that it can be immediately used in simple field laboratories without access to histological equipment. Additionally, the technique can 
be successfully applied independently from the reproductive season. In fact, the experiment was conducted between May and July and therefore outside the normal spawning period for sea bass in the Mediterranean area.

In conclusion, the wet-squash preparations proved to be a practical and effective means of rapidly determining the sex of juvenile sea bass. Consequently, they may replace labour-intensive sectioning and staining in large experimental applications and particular field studies. In many respects, the technique herein described will enable to considerably shorten experimental experiences concerned with sex control in this species and to provide rapid access to sex ratios information.

\section{Acknowledgements}

Christian Fauvel is gratefully acknowledged for his collaboration. The authors are grateful to Jennifer Aïm, Stéphanie Fleur, Maxime Laye and Lionel Zamora for their contribution. The paper by Saillant et al. is cited with the author's permission.

\section{References}

Abucay, J.S., Mair, G.C., Skibinski, D.O.F. \& Beardmore, J.A. (1999). Environmental sex determination: the effect of temperature and salinity on sex ratio in Oreochromis niloticus L. Aquaculture 173, 219-234.

Baras, E., Jacobs, B. \& Mélard, C. (2001). Effect of water temperature on survival, growth and phenotypic sex of mixed $X X-X Y$ progenies of Nile tilapia, Oreochromis niloticus. Aquaculture 192, 187-199.

Barnabé, G. (1976). Contribution à la connaissance de la biologie du loup, Dicentrarchus labrax L. (Serranidae) de la région de Sète. Thèse Doct. D’État, Université du Languedoc, Montpellier, 426 pp. 
Chatain, B., Saillant, E. \& Peruzzi, S. (1999). Production of monosex male populations of European sea bass, Dicentrarchus labrax L., by use of the synthetic androgen $17 \alpha$-methyldehydrotestosterone. Aquaculture 178, 225-234.

Gorshkov, S., Gorshkova, G., Knibb, W. \& Gordin, H. (1999). Sex ratios and growth performance of European sea bass (Dicentrarchus labrax L.) reared in mariculture in Eilat (Red Sea). The Israeli Journal of Aquaculture - Bamidgeh $\mathbf{5 1}$ (3), 91-105.

Guerrero, R.D. \& Shelton, W.L. (1974). An aceto-carmine squash method for sexing juvenile fishes. The Progressive Fish-Culturist 36, 56.

Koumoundouros, G., Pavlidis, M., Anezaki, L., Kokkari, C., Sterioti, A., Divanach, P. \& Kentouri, M. (2002). Temperature sex determination in the European sea bass, Dicentrarchus labrax (L., 1758) (Teleostei, Perciformes, Moronidae): critical sensitive ontogenetic phase. Journal of Experimental Zoology 292, 573-579.

Pavlidis, M., Koumoundouros, G., Somarakis, S., Divanach, P. \& Kentouri, M. (2000). Evidence of temperature-dependent sex determination in the the European sea bass (Dicentrarchus labrax L.). Journal of Experimental Zoology 287, 225-232.

Saillant, E., Chatain, B., Menu, B., Fauvel, C., Vidal, M.O. \& Fostier A. Sexual differentiation and juvenile intersexuality in the European sea bass, Dicentrarchus labrax. Journal of Experimental Zoology (In press).

Zanuy, S., Carrillo, M., Felip, A., Rodríguez, L., Blázquez, M., Ramos, J. \& Piferrer, F. (2001). Genetic, hormonal and environmental approaches for the control of reproduction in the European sea bass (Dicentrarchus labrax L.). Aquaculture 202, 187-203. 